\title{
Study on the Influence of High Speed Railway Planning and Construction on Linyi City and Policy Adjustment
}

\author{
Zhongxiu Liu ${ }^{1}$ \\ ${ }^{1}$ School of civil engineering and architecture, Linyi University, Linyi, Shandong 276000, China \\ *Corresponding author.Email: liuzhongxiu@lyu.edu.cn
}

\begin{abstract}
High speed railway brings opportunities and challenges to the development of the city. Linyi plans to build a number of high-speed railways, of which Lunan high-speed railway has been opened to traffic by the end of November 2019. In the future, the Beijing to Shanghai Second Line will connect with Lunan high speed railway, Jilai high speed railway extension line and Linlian high speed railway to build a "Mi" type railway hub in Linyi. After the opening of high-speed railway in Linyi, how to play a good effect and reduce the adverse effects are proposed.
\end{abstract}

Keywords: high speed railway, planning and construction, influence, policy adjustment, Linyi city

\section{INTRODUCTION}

With the development of economy, the construction of high-speed rail is booming. High speed rail has become an important urban infrastructure and one of the main modes of transportation. High speed rail not only brings convenience to people's life, but also promotes the development of the station city economy. In 1964, the first high-speed rail in the world was completed and opened to traffic in Japan. The opening of Qinhuangdao to Shenyang passenger dedicated line in 1999 marks the opening of high-speed railway construction in China [1]. With the development of the last two decades, China has become the country with the strongest integration ability, the most comprehensive technology, the longest operating mileage and the largest scale under construction. The high-speed railway network greatly shortens people's travel time, becomes one of the most important means of transportation, and also brings opportunities and challenges for the development of the city.

\subsection{Research Background}

Linyi City, with a total area of 17191.2 square kilometers and a total population of 11.619 million, is located in the logistics city in the southeast of Shandong Province. It is the central city in the southeast of Shandong Province, with the largest population and area in Shandong Province, and is also a key development city of the state. Linyi is planning to build a number of high-speed railways, of which Lunan high-speed railway (the section from Linyi to Qufu of Rilan high-speed railway) will be opened to traffic by the end of November 2019, and Linyi will officially enter the era of high-speed railway. In the future, the Beijing Shanghai second line will connect with the Lunan high speed railway, the extension line of Jinan Lai high speed railway and Linlian high speed railway, build a "Mi" type railway hub in Linyi, strengthen the close connection between Linyi and major cities, and promote the economic development of Linyi. In the future, Linyi will arrive in Jinan in one hour, Qingdao in two hours, Beijing and Shanghai in three hours. The opening of high-speed rail is both an opportunity and a challenge. Linyi City has great development potential. The planning and construction of high-speed railway in Linyi city will inevitably have an important impact on the surrounding areas of Linyi city.

\subsection{Research Meaning}

With the rapid development of high-speed rail, many cities are connected in series, and the flow of people, money and things between cities and regions is faster, which promotes the economic development of cities along the line, effectively shortens the time distance between cities along the line, greatly improves the convenience of external transportation [2], and also affects the surrounding areas of cities.

The impact of high-speed rail on the city is not only from the formal operation of high-speed rail, but also from the introduction of high-speed rail planning. The planning and construction of high-speed rail provides a huge development opportunity for the development of Linyi city. The development of high-speed rail has brought many impacts on the development of cities along the line, including increasing accessibility, promoting the adjustment of urban spatial structure, industrial structure, regional economic development, development around stations, employment population promotion and other favorable aspects, as well as the siphon effect of large cities and other adverse aspects. As a third tier city, how to play a good role and weaken the adverse effects after the opening of high-speed rail in Linyi, this study will build the high-speed rail area into a new engine of economic development in Linyi to provide suggestions and countermeasures. 


\section{PLANNING AND CONSTRUCTION OF LINYI HIGH SPEED RAILWAY}

\subsection{Lunan High Speed Railway}

2014In April 2004, the national development and Reform Commission officially approved the inter city rail transit network planning (adjustment) of Shandong Province in the Bohai Rim region. It agreed to build the Lunan high speed railway, which runs through Linyi from the east to the west, passing through Linyi City, Qufu City and Jining City, and has 17 stations, including lijiazhai station, Junan north station, Linyi north station, Feixian north station and Mengshan stationt. After the completion of Lunan high speed railway, it will be interconnected with Beijing Shanghai high speed railway, Qingdao Lianyungang railway, Zhengzhou Xuzhou passenger dedicated railway, the planned Beijing Jiulong passenger dedicated railway and Beijing Shanghai second channel five national trunk railways. Linyi will enter a new era of high-speed rail, which is of great significance to promote the transformation of new and old driving forces and the sustainable and healthy economic and social development along the line. 2014In 2016, the construction of Linyi Qufu section of Lunan high speed railway was started. It is scheduled to open to traffic on November 20,2019, a year and a half ahead of schedule. Its construction will improve the travel efficiency of people in southern and southwestern Shandong, help to expand and deepen cooperation and exchanges with Central Plains Economic Zone and other regions, help southern Shandong to efficiently and conveniently undertake the industrial transfer of Beijing Tianjin Hebei Economic Zone and Yangtze River Delta Economic Zone, and give full play to the industrial advantage leading and radiation leading role of the economic developed zone.

2016Linyi became one of the railway hubs in 50 key areas of the country. Linyi north railway station is located as an important station of Jinan Railway Bureau, a regional largescale hub station and the core hub station of Lunan high speed railway. After completion, Linyi north railway station will become the third largest high-speed railway station in Shandong Province, the first-class comprehensive transportation hub of modern high-speed railway in China and the largest one at Prefecture and city level. Build a new three-dimensional traffic mode of high-speed rail, intercity, subway, urban public transport and taxi, and realize seamless docking and zero distance transfer.

\subsection{Second Line of Beijing Shanghai High Speed Railway}

The second line of Beijing Shanghai high speed railway, also known as the second channel of Beijing Shanghai high speed railway, is the second vertical and the second line of Beijing Shanghai high speed railway. On July 20, 2016, the State Council made a medium and long-term plan for the national high-speed railway, announcing that the second channel of Beijing Shanghai high speed railway will reach Lanling through Yishui, Yinan, Hedong and Lanshan in Linyi City and finally pass through Linyi. It is planned that the second line of Beijing Shanghai high speed railway and Lunan high speed railway will be incorporated into the North Station of Linyi high speed railway and build a comprehensive hub of the North Station of Linyi high speed railway. The second line of Beijing Shanghai high speed railway has two stations in Linyi City: Yimeng station and Linyi north station. Yimeng station is a common station of Yishui County, Yinan county and Ju county. Yimeng station is located to the east of beichangshen expressway, Shilipu Town, Yishui County. After the completion of the second line of the Beijing Shanghai high speed railway, it can relieve the pressure of the existing high speed railway transportation capacity tension, connect the Beijing Tianjin Hebei, Bohai rim, Huaihai economic belt and the Yangtze River Delta, and promote the economic growth and social development of the areas along the line.

\subsection{Jinan-Laiwu-Linyi High Speed Railway}

The design speed of Jinan lailin high speed railway is 350 $\mathrm{km} / \mathrm{h}$, which passes through Jinan East, Zhangqiu District, Laiwu District, Xintai City, Mengyin County and Linyi city.On December 29, 2017, the construction of Jilai highspeed railway began. It is expected to open to traffic in 2020 forming a high-speed railway channel running through Linyi city. The Jilai high-speed railway will extend to the south, enter Linyi city through Mengyin, and connect with the North Station of Linyi high-speed railway through Lunan high-speed railway. The high-speed railway in Jilai can be connected with the Beijing Shanghai high speed railway and the second line of Beijing Shanghai high speed railway, which can supplement the three-dimensional traffic in Linyi, the high-speed railway network in Linyi, make the high-speed railway network more intensive and residents travel more efficiently in the middle of the road.

\section{ANALYSIS AND PREDICTION OF HIGH SPEED RAILWAY ON THE DEVELOPMENT OF LINYI CITY}

\subsection{Impact at the Macro Level}

\subsubsection{Increase accessibility}

After the opening of high-speed railway, the accessibility of cities with stations along the line has been improved, and the economic ties between cities have become closer, with the second tier cities having the most significant impact [3].Zhong Yexi [4] and others believe that there is a gap in the improvement of urban accessibility, the central city is significantly improved, and the development of high-speed 
rail network is gradually catching up with the impact of urban spatial location on the level of urban accessibility. Song Wenjie [5] thinks that the accessibility of big cities is improved, but some small and medium-sized cities even lead to their accessibility lower than before the opening of high-speed rail.It can be seen that experts agree that the impact on accessibility is obvious in large cities, and there are differences in small and medium-sized cities.

First, the opening of high-speed rail enriches people's choice of travel mode and shortens the space perception distance. Linyi takes 1 hour to Jinan and Xuzhou, 2 hours to Qingdao, 3 hours to Beijing, Shanghai, Xi'an, 6 hours to Lanzhou and Xining, and most days to Urumqi and other cities. Linyi will enter one hour commuter circle, two hour business circle of major city groups and three hour traffic circle among major cities in China. Secondly, the opening of high-speed rail improves the location advantage of the city. The high-speed railway has brought great convenience for the acceleration of the flow of people, logistics, capital and information, and provided new choices, strengthening the connection between Linyi City and Beijing Tianjin Hebei, Yangtze River Delta and Bohai Economic Belt. It provides the basis for undertaking the relevant urban industrial transfer, industrial structure adjustment, regional economic development, etc. Finally, the radiation effect of the opening of high-speed rail promotes the development of towns and villages along the line to the high-speed rail line, promotes the development of towns along the line and improves the level of urbanization.

Ding Jiayu [6] thinks that the most competitive travel time of high-speed rail is 1.5-3 hours. Less than 1.5 hours, the convenience and economy of cars and ordinary trains are dominant; more than 3 hours, aircraft efficiency is dominant. Linyi's industrial hinterland will be mainly within the threehour metropolitan area, namely Beijing Tianjin Hebei and the Yangtze River Delta.

\subsubsection{Regional economic development}

The construction and operation of high-speed railway promotes the economic development of the cities along the line, and the development of regional economy provides power for the development of high-speed railway. The whole life cycle of high-speed railway construction and operation can promote regional economic development. Before the construction of high-speed railway, increase the investment layout, promote the investment of related industries during the construction, and increase the economic exchanges between regions to promote economic growth after the completion [7].Qin summed up the direct and indirect effects of high-speed rail on regional economic growth. The direct effect is investment pulling effect, and the indirect effect is that high-speed rail affects regional economic growth by improving traffic and regional interaction. Whether the opening of high-speed rail can promote regional economic growth depends on the size of "spillover effect" and "agglomeration effect" [8].

Taking Lunan high speed railway as an example, the investment is more than 75 billion, affecting the population of Rizhao, Linyi, Zaozhuang, Jining and Heze 32 million along the line. With a large population and complementary industries, the opening of high-speed rail in southern Shandong will promote the coordinated development of the economic belt and create a new growth pole of Shandong economy. As a regional center city, Jining Linyi in the middle of China drives the coordinated development of Rizhao and Heze on the East and West wings. South Shandong high speed railway connects Zhengzhou in the West and promotes the development of the connection between the South Shandong economic belt and the Central Plains city group. The construction of high-speed railway has strengthened the connection between Linyi City, Beijing Tianjin Hebei, Yangtze River Delta and other urban agglomerations, which is conducive to strengthening the flow of production factors and optimizing the industrial structure.

\subsubsection{Promoting urban economic growth}

The impact of high-speed rail on urban economic growth is very obvious, and some scholars have made corresponding explanations from accessibility, population flow, urban connection function, urban housing price, employment effect, industrial structure and industrial development [8].

According to the data released by Shandong Provincial Bureau of statistics, by 2018, Linyi's industrial, urbanization level and residents; consumption capacity were lower than the provincial average. Linyi high speed railway construction and operation, to help the city's economic development.

\subsection{Impact at the Meso Level}

\subsubsection{Industrial layout and adjustment optimization}

After the opening of the high-speed railway, Linyi logistics, tourism, high-tech industries, high-end services and other industries will be greatly promoted. Linyi is the capital of national logistics and a famous city of Commerce and trade. In 2017, Linyi completed a total logistics volume of 2.8 trillion yuan, second only to Qingdao, ranking second in Shandong Province. In 2016, Linyi became one of the railway hubs in 50 key areas of the country. In 2019, Linyi was selected into the list of national logistics hub construction and positioned as a business service type. The original railways, highways and airlines in Linyi are far from meeting the needs of economic development. After the opening of high-speed railway, people flow, logistics, capital flow and information flow have a more efficient and safe choice.

The changes of tourism in the opened high-speed railway areas are as follows: promoting the development of tourism industry in the end cities and cities along the line, regional tourism cooperation, upgrading and transformation of 
tourism system, etc. Linyi has realized that the opening of high-speed rail will provide a great opportunity for Linyi citizens to travel and attract foreign tourists. On the eve of the opening of high-speed rail, we will go to Beijing, an important tourist market, for tourism promotion and exhibition. To publicize the image of "Colorful Yimeng" as a tourist city, to launch special cultural tourism line products, to attract more tourists to climb Mount Meng, listen to Yimeng minor, taste Linyi delicacies and experience the traditional culture of Yimeng.[9]

With the increasingly perfect urban functions of Linyi, more high-tech industries and high-end service industries will migrate from developed areas to gather in Linyi, and further strengthen the coordinated development of Linyi, Beijing, Tianjin, Hebei and the Yangtze River Delta [10].

The construction of high-speed rail transport infrastructure provides good conditions for the development of tertiary industry. In 2017,2018 and in the first half of 2019 the proportion of added value of the three industries in Linyi was 8.3:43.4:48.3, 7.8:43:49.2, 7.7:42.7:49.6 (source: Linyi statistical information network), and the proportion of the third industry increased steadily. After the high-speed opening to traffic, the huge flow of people will bring a large number of catering, shopping, consumption, leisure and service demands, provide more employment opportunities, drive the development of logistics, exhibition, finance, commerce, catering, public entertainment and other industries, as well as tourism and real estate industries, and create a new important economic growth point in Linyi.

\subsubsection{Adjustment of urban spatial structure}

The research on the impact of high-speed rail on urban spatial structure can be summarized as siphon effect, filtration effect and urbanization effect [11].The siphon effect will make capital, talents and so on gather in big cities, and Linyi may face the dilemma of some enterprises moving out and brain drain. The filtering effect is reflected in the competition with Beijing, Shanghai, especially Jinan and Qingdao. Linyi is at a disadvantage, the economic growth rate slows down, or it is replaced by other cities in regional competition, and the gap with high-level cities is further increased. The urbanization effect is due to the change of travel time caused by high-speed rail, which makes people work and live in different places, but actually feel in the same city. The same city effect will appear in the county or Jinan, Qingdao, Linyi work or the opposite situation.

High speed rail affects the functional division of the city, changes the way of urban land use, and promotes the formation of urban system [12].Linyi north station is located at the intersection of Yimeng Road and Xiaosheng Street, Baishabu Town, Lanshan District, Linyi City, Shandong Province, and close to the north of Beicheng New Area. After completion, it will become a three-dimensional new traffic hub of Linyi, a new power for the secondary center and North expansion of Beicheng New Area. Linyi high speed railway station area is planned to be a high-speed railway hub cultural town, realizing the functions of
Commerce, finance, logistics, culture, etc. The high-speed rail area is located in the edge area of the Beicheng New Area of Linyi city. The land price is relatively cheap. After completion, the land for road traffic and facilities, commercial and residential use has increased, becoming a new functional mixed area. Around the high-speed rail station, logistics, commerce, health care and other industries can be developed to change the original urban land structure. There are three counties and many small towns along Linyi section of Lunan high speed railway, namely Fei County, Pingyi County and Junan County. The planning and construction of high speed railway will attract more industries and population to develop along the line, especially after opening to traffic, it will cause the adjustment of industrial structure and the expansion of urban scale along the line, and promote the adjustment and improvement of the scale structure of Linyi Urban system.

\subsubsection{Population in the region}

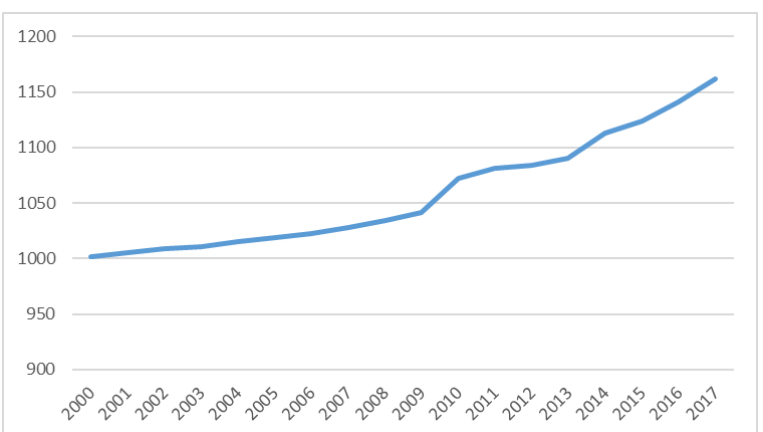

Figure 1 total population of Linyi City from 2000 to 2017 (data source: Linyi Statistical Yearbook)

It can be seen from the figure 1that the population of Linyi city increased year by year from 1978 to 2017 . As of 2017 , the total population of Linyi City reached 11.619 million, and the level of urbanization reached $57.6 \%$. In 2018, the employment of Linyi city increased by 124000 , and the total unemployment rate of the whole year was within $2.27 \%$.In April 2019, the state issued a notice that cities with a permanent population of 3-5 million in urban areas will release the conditions for settling down of rural population and migrant population. Cities with a permanent population of 1-3 million in urban areas will no longer set the conditions for settling down. The permanent population of Linyi city is no more than 3 million. There is no restriction on the settling down of rural households and house purchasers of migrant population. In 2018, Linyi city will realize a total of agricultural people. As a result, it can attract foreigners to purchase houses and settle down in Linyi and attract rural residents to enter the city. For every additional high-speed rail station City in Shandong Province, the number of employees in the province will increase by 620.6 million [13].Especially after the completion and opening of the high-speed railway, the cities and towns along the high-speed railway will gather new industries, provide jobs and attract more employment and 
living population. Relevant research [14] shows that the construction of high-speed rail has a significant attraction to the population of large cities, but it will accelerate the population outflow of small cities. Linyi should also pay attention to the possibility of some people moving to Jinan, Qingdao or even Beijing and Shanghai after the completion of the high-speed railway.

\subsection{Impact at the Micro Level}

The impact of the micro level is mainly reflected in the re planning and development of the site surrounding areas. The urban high-speed railway station area has a catalytic effect on its surrounding space, which leads to the transformation of the area and the reorganization of urban space [11].Linyi high speed railway area is now positioned as one of the railway transportation hubs in Shandong Province, a green industry cluster led by modern service industry and a new urbanization demonstration area with urban-rural integration. Greenland Group plans to invest 50 billion yuan in the construction of China's Linyi high speed rail intercity space station project in Linyi, to build five characteristic areas, including the core area of high-speed rail, the ancient charm and new wind area, the ecological livable area, the elderly care and leisure area, and the rural leisure area, which are divided into four parts: production, education and research, tourism and entertainment, pension, and medical and health care.

The construction of Characteristic Towns in Linyi is combined with the construction of high-speed railway stations. Four high-speed railway towns are built in Lingang District, Junan, Feixian County and Baishabu Town in Beicheng New Area. Lingang district relies on Lijiazhai station to build a high-speed railway dream town. Junan North Station characteristic town plans to build a characteristic industrial park dominated by tourism, commerce and characteristic industries. Fei County should build a "high-speed railway hub, cultural town" development ideas. The high-speed rail area of Linyi north station is integrated with the planning of Baishabu Town, with the positioning of high-speed rail hub, the integration of industry and city, and the leading industries of Commerce and intelligent logistics.

\section{COUNTERMEASURES AND SUGGESTIONS FOR THE DEVELOPMENT OF LIINYI HIGH SPEED RAILWAY}

Through the above analysis and prediction, high-speed rail is a double-edged sword, with opportunities and challenges. The high-speed rail has increased the accessibility of the city, relied on the support of regional economy for the development of the surrounding areas of the city, and attracted high-end industries such as service industry. How to take advantage of the opportunity of high-speed rail and the position of railway hub, develop the urban economy and seize the commanding point of regional cities in Linyi, and put forward the countermeasures and suggestions for the development of high-speed rail in the surrounding areas of the city.

\subsection{Regional Economy: Give Full Play to its Characteristics, Lead the Economic Belt of Southern Shandong, and Integrate into Beijing Tianjin Hebei and the Yangtze River Delta.}

Generally, we think that the surrounding area of high-speed railway station will become a new engine of urban development. The reality is that the regional economic development will affect the development of the surrounding area of high-speed railway station ${ }^{[6]}$. So we should change our ideas and seize the opportunity of high-speed rail. The high-speed railway construction planning of Linyi city provides a more convenient and efficient way to travel, which is conducive to giving full play to the advantages of low cost and high efficiency of Linyi trade logistics industry. Under the favorable policies of national level logistics hub construction, the trade service-oriented logistics will have a greater development. This is also conducive to Linyi to continue to play the leading role of regional central cities in the Lunan Economic Belt.

The economic diffusion and agglomeration brought by high-speed rail coexist. After the opening, within the threehour economic circle which is the most affected, it will promote regional economic complementarity, resource sharing and advantage complementarity, actively undertake the industrial transfer and spillover of Beijing, Tianjin, Hebei and the Yangtze River Delta, participate in the regional industrial adjustment in a wider range, optimize its industrial structure, and transform and upgrade its economic structure. High speed rail provides good conditions for the development of tertiary industry. Linyi develops transportation, catering, accommodation, business, tourism and other industries in and around the high speed rail.

\subsection{Seize the Opportunity of Population Flow and Improve the Level of Urbanization}

In 2018, the permanent population of Linyi city was 10.624 million, ranking first in the province, with a urbanization rate of $51.54 \%$, the second lowest in the province. The level of urbanization was low, with great potential for future development. The planning and development of high-speed rail construction and surrounding areas will attract people to gather along the high-speed rail, bringing new development opportunities for urbanization. At the same time, through policy guidance, improve business environment to attract more foreign investment and industrial settlement in Linyi. Attract more employment through industrial development. 
Planning Society: China Urban Planning Society,

\subsection{Improve the Image of the City and Adjust the Layout of the City Planning}

With the opening of high-speed rail, the accessibility outside the city has improved, and people's perception of space distance has shortened. If the high-speed rail transfers for more than 10 minutes, the city's accessibility experience will decline. In the planning and design of high-speed railway station, it is necessary to pay attention to the transfer experience of passengers, and plan to improve the exit of long-distance passenger transport, city bus, taxi, subway, etc. Linyi high speed railway station has become a new transportation hub of the city, and the surrounding areas are planned to build logistics, wood industry, health care, housing, etc. which will adjust the layout of urban planning and optimize the urban structure.

\section{ACKNOWLEDGMENT}

This work was supported by Linyi Federation of Social Sciences (2019LX062) and Shandong soft science (2019RKB01464)

\section{REFERENCES}

[1] Wang jiaoe, Ding Jinxue. Study on the impact of high-speed railway on urban spatial structure in China [J]. International urban planning, 2011, 26 (6): 49-50

[2] Chu Nanchen, Jiang Bo, Li Xiaoqing, et al. Analysis of generalized transportation cost and land value increment along Harbin Dalian high speed railway [J]. Transportation system engineering and information, 2016,16 (2): 19-24

[3] Wen Yi, Han Xu. The impact of high-speed rail on China's Urban Accessibility and regional economic spatial pattern [J]. Human geography, 2017,32 (01): 99108

[4] Zhong Yexi, Huang Jie, Wen Yuzhao. Analysis of the impact of high-speed rail on the reachable character of Chinese cities [J]. Geosciences, 2015,35 (04): 387-395

[5] Song Wenjie, Zhu Qing, Zhu Yuemei, Kong Cuicui, Shi Yujin, Gu Yongtao. The impact of high-speed rail on the development of different scale cities [J]. Economic geography, 2015,35 (10): 57-63

[6] Ding Jiayu. Research on the impact of high-speed railway passenger transport hub on the development of urban surrounding areas [a]. China Urban Planning Society. Urban times, collaborative planning - 2013 China urban planning annual conference proceedings (01 - Urban Road and traffic planning) [C]. China Urban
2013:10

[7] Guo Rong, He Yongjun. Study on the impact of high speed rail on regional urban economic development [J]. China's Collective Economy, 2019 (25): 16-17

[8] Qin Chenglin, Huang Longjie. Research progress of high speed rail and regional economic development in China [J]. Journal of Guangxi University (PHILOSOPHY AND SOCIAL SCIENCES EDITION), 2018,40 (05): 72-79

[9] Wang Degan, Chen Tian, Li Li, Zhang He. Study on the impact of foreign high-speed railway on tourism and Its Enlightenment [J]. Geosciences, 2012,32 (03): 322328

[10] Li Tao, Zhang Peng, Sun Chengxiu, Liu Xiao. Analysis of the impact of high-speed rail on the economic development of Linyi City and countermeasures and suggestions $[\mathrm{J}]$. Vision of continental bridge, 2016 (12): 51-56

[11] Xu Yinfeng, Wang Degen. Research progress and Prospect of high-speed rail effect of urban spatial structure in China $[\mathrm{J}]$. Progress in geographic science, 2018,37 (09): 1216-1230

[12] Qin Haoyuan. Study on the influence of Lunan high speed railway on the spatial structure of Linyi City [D]. Qufu Normal University, 2016

[13] Wang Junli. Study on the impact of the opening of high-speed rail on the regional economic growth of Shandong Province [D]. Jilin University, 2019

[14] Li Jing. Heterogeneity of the impact of high-speed rail construction on population mobility [D]. Shandong University, 2019

[15] Li Shiqing. Research on the correlation between the development of the surrounding areas of high-speed railway station and the urban development [a]. China Urban Planning Society. Diversity and inclusiveness 2012 China urban planning annual conference proceedings (05. Urban road and traffic planning) [C]. China Urban Planning Society: China Urban Planning Society, 2012:15 